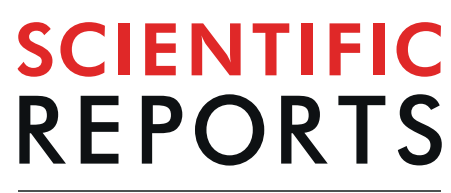

natureresearch

\title{
OPEN Transmural and rate-dependent profiling of drug-induced arrhythmogenic risks through in silico simulations of multichannel pharmacology
}

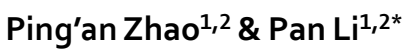

In vitro human ether-à-go-go related gene (hERG) inhibition assay alone might provide insufficient information to discriminate "safe" from "dangerous" drugs. Here, effects of multichannel inhibition on cardiac electrophysiology were investigated using a family of cardiac cell models (Purkinje (P), endocardial (Endo), mid-myocardial (M) and epicardial (Epi)). We found that: (1) OT prolongation alone might not necessarily lead to early afterdepolarization (EAD) events, and it might be insufficient to predict arrhythmogenic liability; (2) the occurrence and onset of EAD events could be a candidate biomarker of drug-induced arrhythmogenicity; (3) M cells are more vulnerable to drug-induced arrhythmias, and can develop early afterdepolarization (EAD) at slower pacing rates; (4) the application of quinidine can cause EADs in all cell types, while $I_{\mathrm{NaL}}$ is the major depolarizing current during the generation of drug-induced EAD in $P$ cells, $\mathrm{I}_{\mathrm{CaL}}$ is mostly responsible in other cell types; (5) drug-induced action potential (AP) alternans with beat-to-beat variations occur at high pacing rates in $\mathrm{P}$ cells. These results suggested that quantitative profiling of transmural and rate-dependent properties can be essential to evaluate drug-induced arrhythmogenic risks, and may provide mechanistic insights into drug-induced arrhythmias.

Drug-induced cardiotoxicity has been a major concern since the early stage of novel drug development. Unexpected post-marketing occurrence of cardiotoxic effects remains a leading cause of drug withdrawal and relabelling ${ }^{1-3}$. As defined by the International Conference of Harmonization Expert Working Group for all drugs in development, QT interval prolongation has been used as a biomarker to predict the potential risk of Torsade de Pointes (TdP) ${ }^{4,5}$. Most drugs that prolong the QT interval inhibit cardiac potassium channels encoded by human ether-à-go-go related gene (hERG); therefore, the level of hERG channel inhibition has been the "gold standard" to predict the TdP risk. However, recent studies suggested that the in vitro hERG blockade assay alone provides insufficient information to accurately discriminate "safe" from "dangerous" drugs ${ }^{6}$. For instance, QT prolongation can be induced by drugs that inhibit other ion channels, such as the slow inward-rectifier $\mathrm{K}^{+}$channel (encoded by KCNQ1 and $\mathrm{KCNE} 1^{7}$ ); the arrhythmia associated with hERG inhibition can be mitigated by concurrent blockade of inward $\mathrm{Na}^{+}$or $\mathrm{Ca}^{2+}$ currents ${ }^{8,9}$. As suggested by Kramer et al. ${ }^{10}$, in vitro assays that evaluate multiple ion channel effects (Nav1.5, Cav1.2 and hERG) may improve the prediction of TdP risks when compared to the hERG assay. More recently, the Comprehensive in vitro Proarrhythmia Assay (CiPA) has been proposed to address the misidentification issue of drug-associated TdP risk based on hERG inhibition and QT prolongation data. This new paradigm is based on integrated assessment of a wider range of ion channel dynamics (including Nav1.5 (peak and late), Cav1.2, hERG, Kv4.3, KCNQ1/KCNE1, Kir2.1) in delayed ventricular repolarization; alterations to this process lead to repolarization instability and arrhythmias ${ }^{11}$. In addition to drug-induced QT prolongation, action potential (AP) triangulation has also been reported as a risk factor for both atrial fibrillation and ventricular arrhythmia ${ }^{12}$. Furthermore, the autonomic nervous system can also play an important role in drug-induced

${ }^{1}$ Center for Public Health Informatics, School of Public Health, Xinxiang Medical University, Henan, P.R. China. ${ }^{2}$ Center for Biomedical Innovation, Yunmai Biomedical Research Institute, Henan, P.R. China. *email: panli@xxmu.edu.cn 

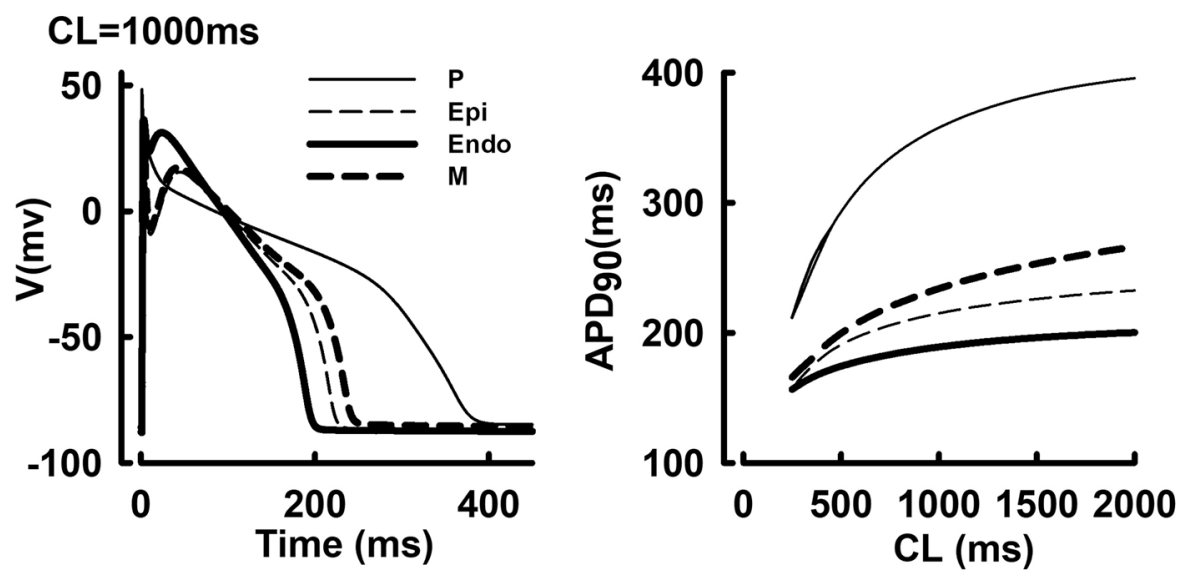

Figure 1. Steady-state Transmural AP morphologies $(\mathrm{CL}=1000 \mathrm{~ms})(\mathrm{A})$ and APD rate adaptation curves $(\mathbf{B})$ in $\mathrm{P}$, Endo, $\mathrm{M}$ and Epi cells.

$\mathrm{TdP}$ risks ${ }^{13}$, and as proposed by Champeroux et al. ${ }^{14}$, the high frequency relationship between magnitude of heart rate and QT interval changes may serve as a new biomarker for assessing TdP liability.

Multi-scale modelling of the heart has been an important tool in advancing our understanding of cardiac excitation-contraction coupling under both physiological and pathological conditions ${ }^{15}$. Recent pharmacological studies have utilized cardiac cell models for the in silico evaluation of drug-induced proarrhythmic risks ${ }^{11,16-18}$. For example, multiple cardiac ion channels were integrated into the human ventricular cell model to improve the assessment of proarrhythmic risk ${ }^{19,20}$. Lancaster et al. proposed a computational approach that combines a human ventricular myocyte model of drug effects and machine learning to evaluate drug-induced TdP risks ${ }^{21}$. Using in silico human endocardial and Purkinje cell models, Le Guennec et al. evaluated how intra- and inter-individual variability can influence drug-induced proarrhythmic effects of hERG inhibition ${ }^{16}$. In addition, tissue models of the heart have also been implemented to quantitatively evaluate drug-induced risk $^{22}$, e.g. Kubo et al. developed a 2-dimensional ventricular wedge preparation model of both non-failing and failing hearts to predict drug-induced QT prolongation and ventricular proarrhythmia ${ }^{23}$.

The mammalian heart functions as a complex biological system ${ }^{24}$, and its emergent properties (e.g. TdP) mostly result from the interplay of sub-system components (e.g. Purkinje cells, and various types of cardiac myocytes across the ventricular wall ${ }^{25}$. Here, we employed a family of in silico cardiac cell models (Purkinje(P), endocardial(Endo), mid-myocardial(M) and epicardial(Epi)) and performed quantitative profiling of drug-induced arrhythmogenic risk at physiological pacing frequencies, to identify potential novel characteristics associated with drug-induced TdP risk, and to provide possible mechanistic insights into drug-specific cardiotoxicity that may facilitating the process of drug development.

\section{Results}

Transmural heterogeneity of AP morphologies and APD rate adaptations. Transmural AP morphologies (steady-state pacing at cycle length $(\mathrm{CL})=1000 \mathrm{~ms}$ ) and AP duration (APD) rate adaptation curves are shown in Fig. 1. The APD in M cells was longer than that in Epi or Endo cells at all CLs, yet was considerably shorter than the APD in P cells. The AP amplitude (APA) in P cells was higher than that in ventricular cells. In Epi and M cells, AP reproduced a characteristic phase-1 notch and dome morphology, which was not apparent in Endo cells. The APD rate adaptation curve is much steeper at short CLs in P cells, compared to ventricular cells. These simulated AP morphologies and APD adaptation curves were consistent with earlier experimental measurements ${ }^{26,27}$.

Drug-induced changes in APD rate adaptations. Drug-induced changes in APD rate adaptations were shown in Fig. 2, with a detailed summary of drug-induced APD prolongation, EAD and AP alternans provided in the Supplemental Table S1. With the application of qunidine, early afterdepolarization (EAD) events were observed in all cell types at all pacing CLs. With the application of ranolazine, sotalol, terfenadine, cisapride, dofetilide or bepridil, EAD events were observed at different CLs, exclusively in M cells. Bepridil can induce EAD at CL $>550 \mathrm{ms,}$ while ranolazine can induce EAD at CL $>1800 \mathrm{~ms}$. Major drug-induced APD prolongation $(>10 \%)$ was observed with the application of bepridil, dofetilide, cisapride or terfenadine, while ranolazine or sotalol caused moderate APD prolongation. With the application of diltiazem or verapamil, major APD prolongation can be observed without EAD events, suggesting APD prolongation alone could be insufficient to trigger EAD. Chlorpromazine or ondansetron caused moderate prolongation without EAD events. In contrast, mexiletine led to minor APD shortening by $2.6 \%$ at $\mathrm{CL}=2000 \mathrm{~ms}$ in P cells, while causing minor APD prolongation in other cell types with no EAD events. In general, the percentages of drug-induced APD prolongations were much higher in M cells. For example, at $\mathrm{CL}=1000 \mathrm{~ms}$, bepridil caused APD prolongation of $15 \%$ (P cells), 27\% (Epi cells) and 25.6\% (Endo cells) respectively, and caused APD prolongation of $158 \%$ in M cells (with EAD). Drug-induced changes in APD is highly celland rate-dependent. For example, drug-induced EAD events are mostly associated with slower pacing rates in $\mathrm{M}$ cells; drug-induced AP alternans mostly occur at fast pacing rates in P cells. 

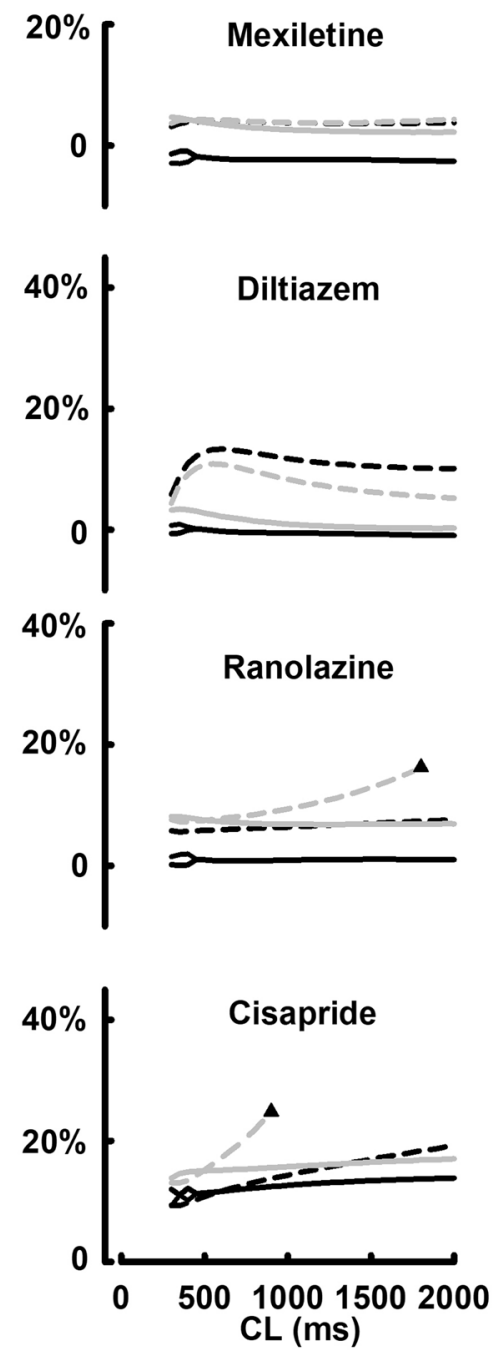

Chlorpromazine

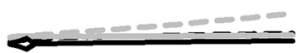

\section{Verapamil}

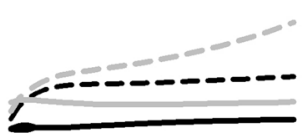

Sotalol
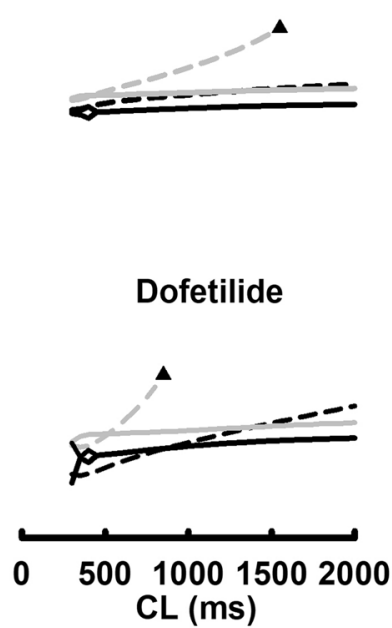

Ondansetron

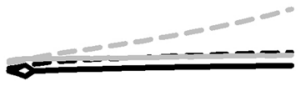

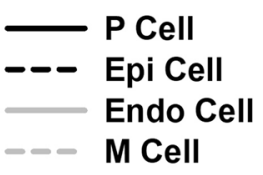

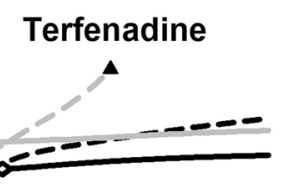

Figure 2. Steady-state drug-induced changes in APD rate adaptations in P, Endo, M and Epi cells. All drugs were applied at their effective free therapeutic plasma concentrations (EFTPCs). Black triangles indicate the onset of EAD events.

Transmural variations of drug-induced arrhythmogenicity. The AP and ionic channel dynamics with the application of diltiazem and mexiletine were shown at $\mathrm{CL}=1000 \mathrm{~ms}$ in Fig. 3. With the application of diltiazem, AP morphology and APD remain largely unaffected despite of a major reduction in $\mathrm{I}_{\mathrm{CaL}}$ in $\mathrm{P}$ cells, while accentuated AP notch and APD prolongation were observed in Epi cells due to decreased $\mathrm{I}_{\mathrm{CaL}}$ and $\mathrm{I}_{\mathrm{Kr}}$. With the application of mexiletine, minor APD shortening or prolongation was evident in P or endo cells respectively. While $\mathrm{I}_{\mathrm{Kr}}$ reduction promotes APD prolongation in both cell types, such effect was mitigated by the concurrent inhibition of $\mathrm{Na}^{+}$channels in $\mathrm{P}$ cells. Transmural variations of drug-induced EAD and AP alternans were show in Fig. 4. With the application of cisapride $(\mathrm{CL}=1000 \mathrm{~ms})$, major reduction in $\mathrm{I}_{\mathrm{Kr}}$ caused APD prolongation by $15.7 \%$ in Endo cells, yet induced EAD in M cells. With the application of dofetilide ( $C L=300 \mathrm{~ms})$, AP alternans was observed at $\mathrm{CL}=300 \mathrm{~ms}$ most due to alternating large-small $\mathrm{I}_{\mathrm{NaL}}$ currents in P cells, while no alternans was shown in $\mathrm{M}$ cells under same conditions.

Rate-dependent variations of drug-induced arrhythmogenicity. AP and underlying ionic channel dynamics at different CLs ( $300 \mathrm{~ms}, 1000 \mathrm{~ms}$ and $2000 \mathrm{~ms}$ ) with the application of chlorpromazine and terfenadine in $\mathrm{M}$ cells were shown in Fig. 5. The effects chlorpromazine exhibit minor rate-dependence with slightly pronounced APD prolongation at $\mathrm{CL}=2000 \mathrm{~ms}$, compared to $\mathrm{CL}=300 \mathrm{~ms}$. With the application of terfenadine, major APD prolongation at $\mathrm{CL}=1000 \mathrm{~ms}(23.6 \%)$ was observed compared to $\mathrm{CL}=300 \mathrm{~ms}$. At $\mathrm{CL}=2000 \mathrm{~ms}$, terfenadine induced $\mathrm{EAD}$ due to the reactivation of $\mathrm{I}_{\mathrm{Ca}}$. Compared to chlorpromazine, terfenadine displays significant rate-denpendence in $\mathrm{M}$ cells under same conditions.

Drug-induced early afterdepolarization (EAD) and AP alternans. With the application of quinidine at $\mathrm{CL}=2000 \mathrm{~ms}$, EAD events were observed in all cell types (Fig. 6). In ventricular cells (Endo, M and Epi), the reactivation of $\mathrm{I}_{\mathrm{CaL}}$ is evident, and it coincides with the EAD upstroke. Particularly, oscillatory EAD events can 


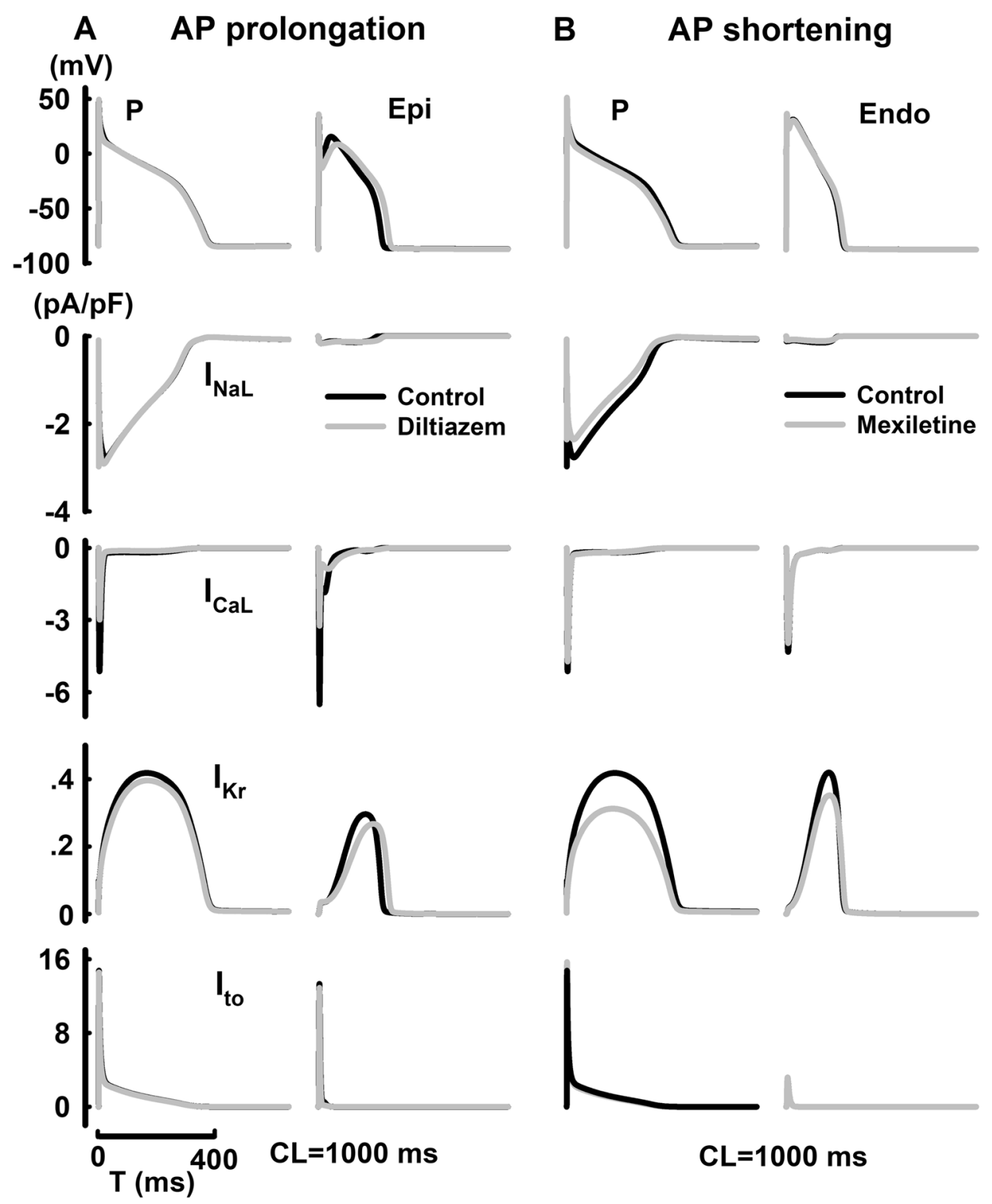

Figure 3. Steady-state cell-dependent AP prolongation, AP shortening and their underlying ionic currents $\left(\mathrm{I}_{\mathrm{NaL}}, \mathrm{I}_{\mathrm{CaL}}, \mathrm{I}_{\mathrm{Kr}}\right.$, and $\left.\mathrm{I}_{\mathrm{to}}\right)$ with the application of diltiazem $(\mathbf{A})$ or mexiletine $(\mathbf{B})$.

been observed in $\mathrm{M}$ cells along with the repetitive reactivation of $\mathrm{I}_{\mathrm{CaL}}$. However, in $\mathrm{P}$ cells, the reactivation of $\mathrm{I}_{\mathrm{CaL}}$ is much smaller in amplitude, and contributes a secondary role in trigger EAD; the EAD upstroke is mostly driven by $\mathrm{I}_{\mathrm{NaL}}$ due to intrinsic electrophysiological heterogeneities between Purkinje and ventricular cells ${ }^{28}$. Such observation is consistent with previous studies ${ }^{28}$. Drug-induced AP alternans and underlying ionic currents were shown in Fig. 7. With the application of dofetilide or bepridil, steady-state AP prolongation with enhanced beat-to-beat variations (AP alternans) was observed at $\mathrm{CL}=300 \mathrm{~ms}$ in P cells; with the application of verapamil, AP prolongation with no beat-to-beat variations was shown ${ }^{28}$. In addition, the application of bepridil can also induce steady-state alternating EAD patterns ( 1 vs 2 EAD upstrokes from beat to beat) at slower pacing rates $(\mathrm{CL}=1050 \mathrm{~ms})$ in $\mathrm{M}$ cells. While $\mathrm{I}_{\mathrm{NaL}}$ is mostly responsible to induce AP alternans in P cells at $\mathrm{CL}=300 \mathrm{~ms}, \mathrm{I}_{\mathrm{CaL}}$ and $\mathrm{I}_{\text {to }}$ play such role in driving AP alternans associated with the EAD morphology in $\mathrm{M}$ cells at $\mathrm{CL}=1050 \mathrm{~ms}$.

\section{Discussion}

In this study, we quantitatively evaluated the transmural characteristics and rate dependence of drug-induced arrhythmogenicity through simulations of multichannel pharmacology using a family of cardiac cell models. Our results reveal diverse cell- and rate-dependent variations of drug-induced arrhythmogenicity, e.g. AP alternans, APD shortening or prolongation, EAD events. For instance, the effects of mexiletine (low risk) exhibit little cell and rate-dependent variations, while significant cell and rate-dependence was observed with the application of bepridil (high risk). In addition, our simulation results suggest QT prolongation alone might be insufficient in predicting TdP risk, since major APD prolongation can be observed with the application of diltiazem or 


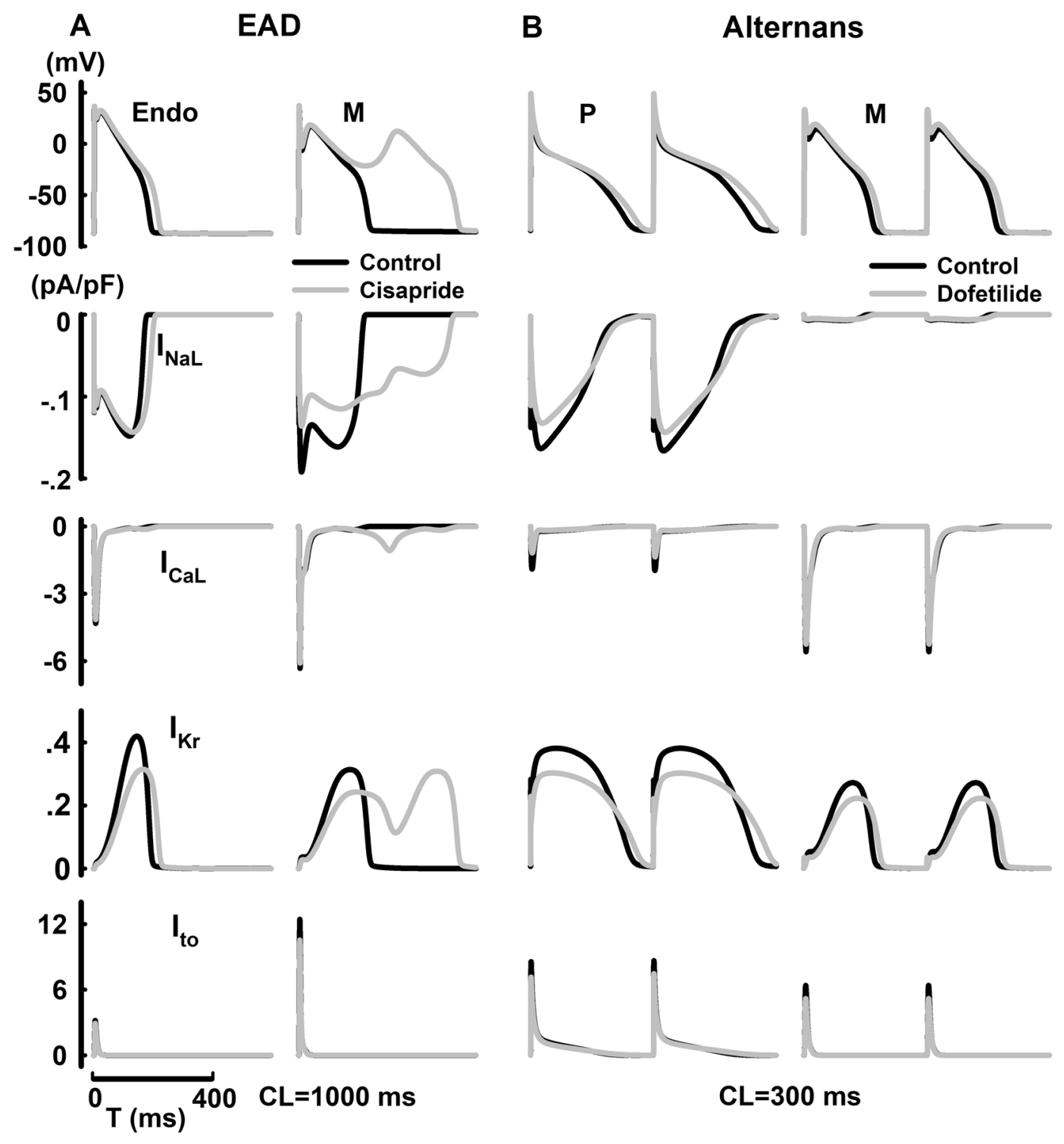

Figure 4. Steady-state cell-dependent $\mathrm{EAD}, \mathrm{AP}$ alternans and their underlying ionic currents $\left(\mathrm{I}_{\mathrm{NaL}}, \mathrm{I}_{\mathrm{CaL}}, \mathrm{I}_{\mathrm{Kr}}\right.$ and $\left.\mathrm{I}_{\mathrm{to}}\right)$ with the application of cisapride (A) or dofetilide (B).

verapamil (low risk) with no EAD events. Veramamil inhibits both $\mathrm{I}_{\mathrm{Kr}}$ and $\mathrm{I}_{\mathrm{CaL}}$ currents, thus it can promote APD prolongation with a smaller $\mathrm{I}_{\mathrm{CaL}}$ current incapable of triggering EAD events. Thus, in addition to QT prolongation alone, it appears that the occurrence and onset of EAD events, especially within physiological pacing window, could be a candidate biomarker for assessing TdP liability. A total of 12 drug were quantitatively evaluated in our study, and only quinidine (high risk) is capable of inducing EAD in all cell types. The predicted high TdP risk of quinidine is consistent with that in previous clinical studies ${ }^{29}$. Our simulation results with ranolazine (low risk) seems to be in conflict with its safety observed in clinical trials ${ }^{30}$, yet it should be noted that ranolazine induced $\mathrm{EAD}$ at $\mathrm{CL}>1800 \mathrm{~ms}$ ( $\sim 33$ beats per minute) in $\mathrm{M}$ cells. Considering electric coupling in the intact ventricular myocardium, ranolazine induced EAD events might occur at very slow and unphysiological pacing rates according to our simulations. However, our results might also indicate possible hidden risks associated with ranolazine. Furthermore, our predictions regarding chlopromazine and ondansetron (minor changes in APD rate adaptation with no EAD events) seem not well in line with their known intermediate risks. This is likely due to their relatively low EFTPC values and minor inhibition effects on ion channels. These results might also indicate possible drug targets other than the CiPA ion channels associated with chlopromazine and ondansetron.

Our simulation results suggest that $\mathrm{M}$ cells, in general, are much more vulnerable to both drug-induced AP prolongation and EAD occurrence, and the intrinsic arrhythmogenicity of $\mathrm{M}$ cells can be much higher than that of other cell types. Despite the controversies surrounding M cells, our results support a major role of the $\mathrm{M}$ cell in promoting pathologic heterogeneities of repolarization ${ }^{31}$. In addition to electric inter-cellular coupling, location and abundance of $\mathrm{M}$ cells in the intact ventricular myocardium could be important determinants to extrapolate our cell simulation results at tissue-level ${ }^{31}$. In addition, we found that drug-induced changes in APD adaptation 


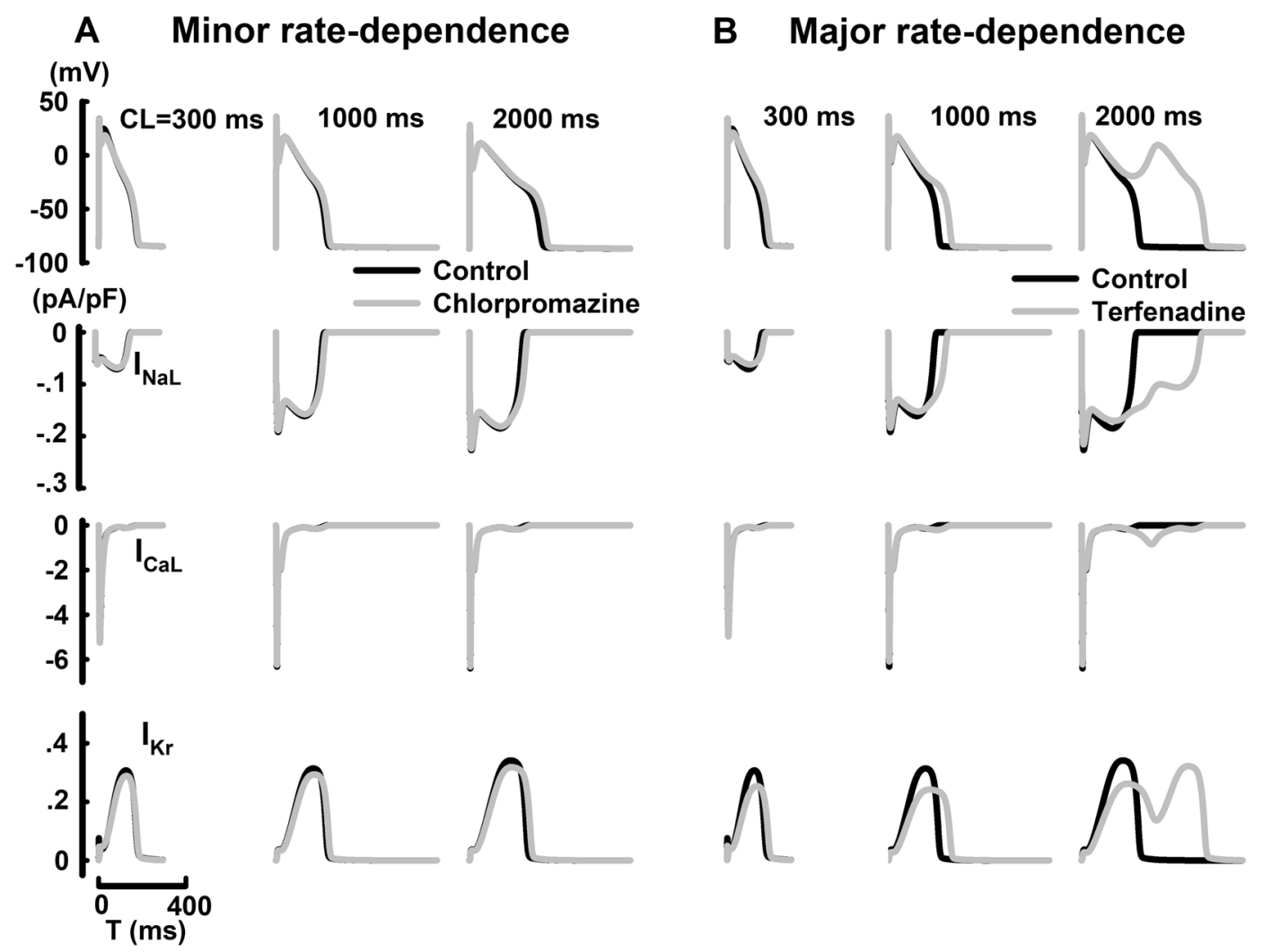

Figure 5. Steady-state rate-dependent variations and their underlying ionic currents $\left(\mathrm{I}_{\mathrm{NaL}}, \mathrm{I}_{\mathrm{CaL}}, \mathrm{I}_{\mathrm{Kr}}\right.$, and $\left.\mathrm{I}_{\mathrm{to}}\right)$ with the application of chlorpromazine $(\mathbf{A})$ or terfenadine $(\mathbf{B})$ in $\mathrm{M}$ cells at different CLs.

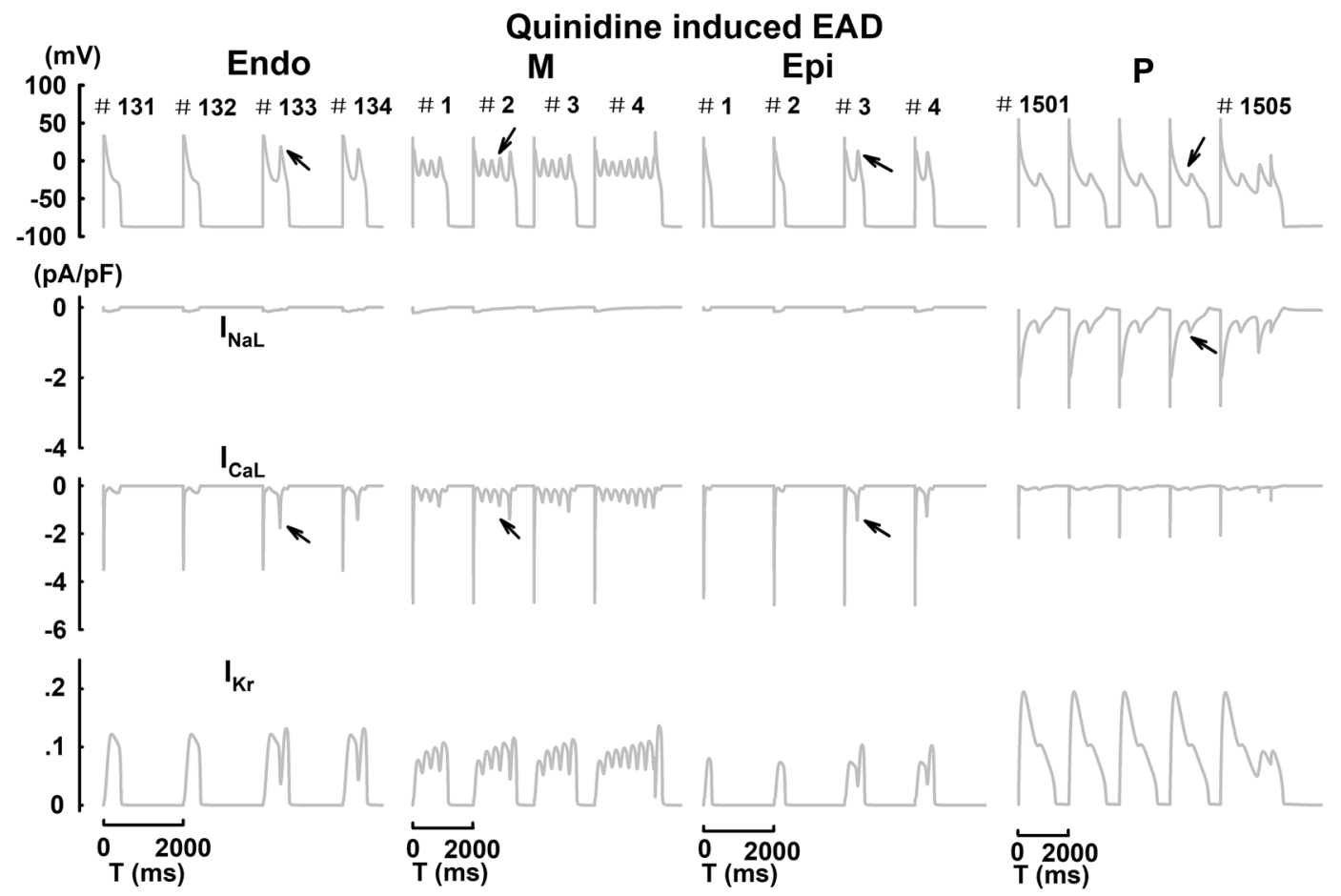

Figure 6. Qunidine induced EAD events and underlying ionic currents in Endo, M, Epi and P cells at $\mathrm{CL}=2000 \mathrm{~ms}$. Black arrows indicate EAD events. 


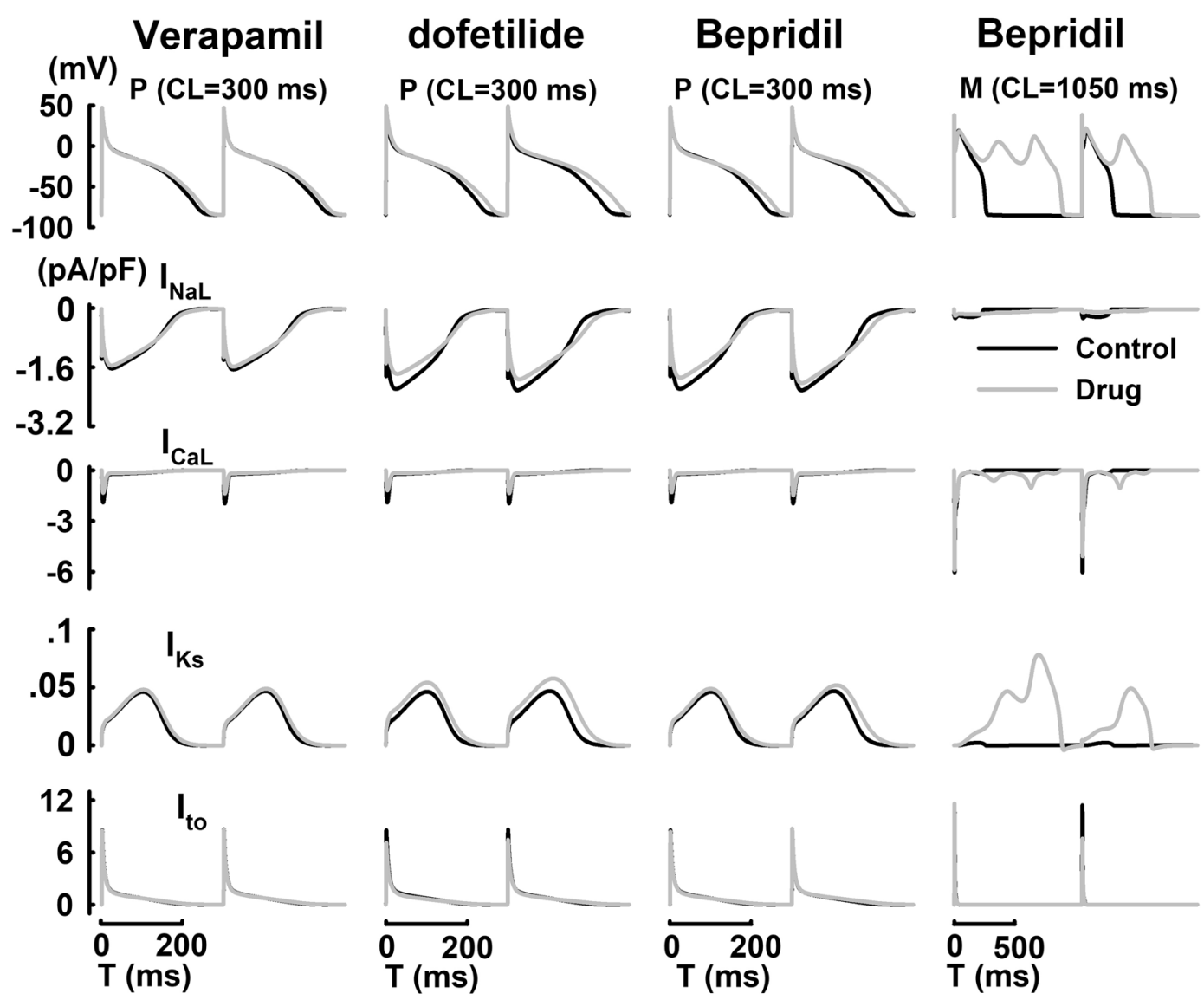

Figure 7. Steady-state AP alternans and underlying ionic currents with the application of verapamil, dofetilide or bepridil at various CLs.

can be important during the evaluation of drug-induced cardiotoxicity. The pronounced increase in APD at slower heart rates allows for the recovery of inactivated $\mathrm{Ca}^{2+}$ or $\mathrm{Na}^{+}$channels, widening the window of EAD generation, a cellular event that can induce $\mathrm{TdP}^{32-35}$. Furthermore, we found that the mechanism of drug-induced EAD generation differs across cell types; EAD generation in $\mathrm{P}$ cells is mostly due to reactivation of $\mathrm{I}_{\mathrm{NaL}}$, while in Endo, $\mathrm{M}$ and Epi cells, $\mathrm{I}_{\mathrm{CaL}}$ plays the predominant role ${ }^{28}$. Intrinsic electrophysiological and ultrastructural heterogeneities between Purkinje and ventricular cells are most responsible for different AP morphologies and ionic mechanisms underlying AP rate adaptation, restitution and EAD events ${ }^{28}$. For instance, $\mathrm{I}_{\mathrm{NaL}}$ in Purkinje cells is a much larger current with distinct decay and recovery kinetics, while $\mathrm{I}_{\mathrm{CaL}}$ is much smaller compared to ventricular cells $^{36-38}$. Purkinje cells are devoid of transverse tubular (T-tubular) network, and exhibits biphasic $\mathrm{Ca}^{2+}$ transient in response to membrane depolarization ${ }^{39}$. Compared to other ventricular cells, $\mathrm{M}$ cells are characterized with prolonged APD and less repolarization reserve mostly due to a larger $\mathrm{I}_{\mathrm{NaL}}$ and a smaller $\mathrm{I}_{\mathrm{Ks}}$, and are therefore predisposed to a higher risk of arrhythmogenesis. In addition, comparing to other cell types, $\mathrm{P}$ cells are generally more sensitive to drug-induced AP alternans at fast pacing rates, that may potentially lead to Purkinje-ventricular conduction abnormalities at tissue/organ level ${ }^{40}$.

We concluded that simulations of multichannel pharmacology in diverse cell types at all physiological pacing rates could be essential to evaluate drug-induced arrhythmogenic risks, and an updated and more comprehensive list of biomarkers associated with cell- and rate-dependent drug responses, instead of hERG inhibition or QT prolongation alone, might improve the prediction of drug-induced cardiotoxicity. Further model development is required to develop human-specific purkinje-ventricular tissue models with spatially distributed intra- and intercellular $\mathrm{Ca}^{2+}$ dynamics, to predict arrhythmogenic risks associated with abnormal $\mathrm{Ca}^{2+}$ handling, such as spontaneous $\mathrm{Ca}^{2+}$ release and delayed after-depolarization (DAD) ${ }^{41}$.

\section{Methods}

A family of cardiac cell models (P, Endo, M and Epi) were derived from the Purkinje and ventricular cell models published by Li and Rudy ${ }^{28}$ and Decker et al ${ }^{42}$ based on experimental measurements of intrinsic heterogeneities of electrophysiological properties (see Supplemental Table S2). These models were developed to include physiologically based representations of intracellular $\mathrm{Ca}^{2+}$ cycling and major membrane ionic currents specific to each cell types, using similar mathematical modelling and validation approaches ${ }^{28,42}$. Multi-channel inhibition effects of 12 CiPA training compounds with known TdP risk (including quinidine, bepridil, dofetilide, sotalol, chlorpromazine, cisapride, terfenadine, ondansetron, diltiazem, mexiletine, ranolazine and verapamil) were simulated at effective free therapeutic plasma concentration (EFTPC) (see Supplemental Table S3 for details) ${ }^{43}$; a 
more extensive list of compounds with known TdP risk classifications has been reported in previous studies ${ }^{6,20,44}$. Steady-state simulation results are for 60 minutes of pacing at a given CL. APD is determined as APD ${ }_{90}(90 \%$ repolarization). Standard programming language $C$ was used. Forward Euler method with an adaptive time step was used for numeric integration. To compare cell-dependent responses, drug-induced changes in AP adaptation were quantified as the percentage of $\triangle \mathrm{APD}_{90, \text { drug }} / \mathrm{APD}_{90, \text { control }}$.

Received: 3 September 2019; Accepted: 21 November 2019;

Published online: 06 December 2019

\section{References}

1. Gottlieb, S. Antihistamine drug withdrawn by manufacturer. BMJ 319, 7 (1999)

2. Josefson, D. Hay fever drug to be banned by the FDA. BMJ (314:248) (1997).

3. Henney, J. E. Withdrawal of troglitazone and cisapride. JAMA 283, 2228 (2000).

4. Yap, Y. G. Drug induced QT prolongation and torsades de pointes. Heart 89, 1363-1372) (2003).

5. International Conference on Harmonisation; guidance on S7B Nonclinical Evaluation of the Potential for Delayed Ventricular Repolarization (QT Interval Prolongation) by Human Pharmaceuticals; availability. Notice. (Fed Regist ;70:61133-61134) (2005).

6. Redfern, W. et al. Relationships between preclinical cardiac electrophysiology, clinical QT interval prolongation and torsade de pointes for a broad range of drugs: evidence for a provisional safety margin in drug development. Cardiovascular Research 58, 32-45, https://doi.org/10.1016/s0008-6363(02)00846-5 (2003).

7. Osteen, J. D. et al. KCNE1 alters the voltage sensor movements necessary to open the KCNQ1 channel gate. Proc Natl Acad Sci USA 107, 22710-22715, https://doi.org/10.1073/pnas.1016300108 (2010).

8. Antoons, G. et al. Late $\mathrm{Na}^{+}$current inhibition by ranolazine reduces torsades de pointes in the chronic atrioventricular block dog model. J Am Coll Cardiol 55, 801-809, https://doi.org/10.1016/j.jacc.2009.10.033 (2010).

9. Johnson, D. M., de Jong, M. M., Crijns, H. J., Carlsson, L. G. \& Volders, P. G. Reduced ventricular proarrhythmic potential of the novel combined ion-channel blocker AZD1305 versus dofetilide in dogs with remodeled hearts. Circ Arrhythm Electrophysiol 5, 201-209, https://doi.org/10.1161/CIRCEP.111.963025 (2012).

10. Kramer, J. et al. MICE models: superior to the HERG model in predicting Torsade de Pointes. Sci Rep 3, 2100, https://doi. org/10.1038/srep02100 (2013).

11. Sager, P. T., Gintant, G., Turner, J. R., Pettit, S. \& Stockbridge, N. Rechanneling the cardiac proarrhythmia safety paradigm: a meeting report from the Cardiac Safety Research Consortium. Am Heart J 167, 292-300, https://doi.org/10.1016/j.ahj.2013.11.004 (2014).

12. Ellermann, C. et al. Action Potential Triangulation Explains Acute Proarrhythmic Effect of Aliskiren in a Whole-Heart Model of Atrial Fibrillation. Cardiovasc Toxicol, https://doi.org/10.1007/s12012-019-09533-w (2019).

13. Fossa, A. A. The impact of varying autonomic states on the dynamic beat-to-beat QT-RR and QT-TQ interval relationships. Br J Pharmacol 154, 1508-1515, https://doi.org/10.1038/bjp.2008.123 (2008).

14. Champeroux, P. et al. The high frequency relationship: implications for torsadogenic hERG blockers. Br J Pharmacol 173, 601-612, https://doi.org/10.1111/bph.13391 (2016).

15. Kerckhoffs, R. C. et al. Multi-scale modeling of excitation-contraction coupling in the normal and failing heart. Conf Proc IEEE Eng Med Biol Soc 2009, 4281-4282, https://doi.org/10.1109/IEMBS.2009.5332708 (2009).

16. Le Guennec, J. Y. et al. Inter-individual variability and modeling of electrical activity: a possible new approach to explore cardiac safety? Sci Rep 6, 37948, https://doi.org/10.1038/srep37948 (2016).

17. Valentin, J. P. \& Hammond, T. Safety and secondary pharmacology: successes, threats, challenges and opportunities. J Pharmacol Toxicol Methods 58, 77-87, https://doi.org/10.1016/j.vascn.2008.05.007 (2008).

18. Fletcher, K. et al. Novel approaches to assessing cardiac safety-proceedings of a workshop: regulators, industry and academia discuss the future of in silico cardiac modelling to predict the proarrhythmic safety of drugs. Drug Saf 34, 439-443, https://doi. org/10.2165/11591950-000000000-00000 (2011).

19. Mirams, G. R., Davies, M. R., Cui, Y., Kohl, P. \& Noble, D. Application of cardiac electrophysiology simulations to pro-arrhythmic safety testing. Br J Pharmacol 167, 932-945, https://doi.org/10.1111/j.1476-5381.2012.02020.x (2012).

20. Mirams, G. R. et al. Simulation of multiple ion channel block provides improved early prediction of compounds' clinical torsadogenic risk. Cardiovasc Res 91, 53-61, https://doi.org/10.1093/cvr/cvr044 (2011).

21. Lancaster, M. C. \& Sobie, E. A. Improved Prediction of Drug-Induced Torsades de Pointes Through Simulations of Dynamics and Machine Learning Algorithms. Clin Pharmacol Ther 100, 371-379, https://doi.org/10.1002/cpt.367 (2016).

22. Romero, L. et al. In Silico QT and APD Prolongation Assay for Early Screening of Drug-Induced Proarrhythmic Risk. J Chem Inf Model 58, 867-878, https://doi.org/10.1021/acs.jcim.7b00440 (2018).

23. Kubo, T., Ashihara, T., Tsubouchi, T. \& Horie, M. Significance of integrated in silico transmural ventricular wedge preparation models of human non-failing and failing hearts for safety evaluation of drug candidates. J Pharmacol Toxicol Methods 83, 30-41, https://doi.org/10.1016/j.vascn.2016.08.007 (2017).

24. Fischer, H. P. Mathematical modeling of complex biological systems: from parts lists to understanding systems behavior. Alcohol Res Health 31, 49-59 (2008).

25. Wilde, A. A. M., Garan, H. \& Boyden, P. A. Role of the Purkinje system in heritable arrhythmias. Heart Rhythm 16, 1121-1126, https://doi.org/10.1016/j.hrthm.2019.01.034 (2019).

26. Kondo, M. \& Tsutsumi, T. Potassium channel openers antagonize the effects of class III antiarrhythmic agents in canine Purkinje fiber action potentials. Implications for prevention of proarrhythmia induced by class III agents. Jpn Heart J 40, 609-619 (1999).

27. Varro, A., Nakaya, Y. \& Elharrar, V. Effect of antiarrhythmic drugs on the cycle length-dependent action potential duration in dog Purkinje and ventricular muscle fibers. J Cardiovasc Pharmacol 8, 178-185 (1986).

28. Li, P. \& Rudy, Y. A model of canine purkinje cell electrophysiology and $\mathrm{Ca}^{2+}$ cycling: rate dependence, triggered activity, and comparison to ventricular myocytes. Circ Res 109, 71-79, https://doi.org/10.1161/CIRCRESAHA.111.246512 (2011).

29. Bauman, J. L. et al. Torsade de pointes due to quinidine: observations in 31 patients. Am Heart J 107, 425-430, https://doi. org/10.1016/0002-8703(84)90081-4 (1984).

30. Shenasa, M., Assadi, H., Heidary, S. \& Shenasa, H. Ranolazine: Electrophysiologic Effect, Efficacy, and Safety in Patients with Cardiac Arrhythmias. Card Electrophysiol Clin 8, 467-479, https://doi.org/10.1016/j.ccep.2016.02.011 (2016).

31. Wilson, L. D., Jennings, M. M. \& Rosenbaum, D. S. Point: M cells are present in the ventricular myocardium. Heart Rhythm 8, 930-933, https://doi.org/10.1016/j.hrthm.2011.01.026 (2011).

32. Cummins, M. A., Dalal, P. J., Bugana, M., Severi, S. \& Sobie, E. A. Comprehensive analyses of ventricular myocyte models identify targets exhibiting favorable rate dependence. PLoS Comput Biol 10, e1003543, https://doi.org/10.1371/journal.pcbi.1003543 (2014).

33. Weiss, J. N., Garfinkel, A., Karagueuzian, H. S., Chen, P. S. \& Qu, Z. Early afterdepolarizations and cardiac arrhythmias. Heart Rhythm 7, 1891-1899, https://doi.org/10.1016/j.hrthm.2010.09.017 (2010).

34. Weissenburger, J., Davy, J. M. \& Chezalviel, F. Experimental models of torsades de pointes. Fundam Clin Pharmacol 7, 29-38, https:// doi.org/10.1111/j.1472-8206.1993.tb00215.x (1993). 
35. Yan, G. X. et al. Phase 2 early afterdepolarization as a trigger of polymorphic ventricular tachycardia in acquired long-QT syndrome: direct evidence from intracellular recordings in the intact left ventricular wall. Circulation 103, 2851-2856, https://doi. org/10.1161/01.cir.103.23.2851 (2001).

36. Zygmunt, A. C. et al. Larger late sodium conductance in M cells contributes to electrical heterogeneity in canine ventricle. Am J Physiol Heart Circ Physiol 281, H689-97 (2001).

37. Antzelevitch, C. \& Belardinelli, L. The role of sodium channel current in modulating transmural dispersion of repolarization and arrhythmogenesis. J Cardiovasc Electrophysiol 17(Suppl 1), S79-S85, https://doi.org/10.1111/j.1540-8167.2006.00388.x (2006).

38. Li, W. et al. Larger rate dependence of late sodium current in cardiac Purkinje cells: A potential link to arrhythmogenesis. Heart Rhythm 14, 422-431, https://doi.org/10.1016/j.hrthm.2016.11.036 (2017).

39. Boyden, P. A., Pu, J., Pinto, J. \& Keurs, H. E. Ca ${ }^{2+}$ transients and $\mathrm{Ca}^{2+}$ waves in purkinje cells: role in action potential initiation. Circ Res 86, 448-455, https://doi.org/10.1161/01.res.86.4.448 (2000).

40. Boyden, P. A. Purkinje physiology and pathophysiology. J Interv Card Electrophysiol 52, 255-262, https://doi.org/10.1007/s10840018-0414-3 (2018).

41. Orchard, C. H., Eisner, D. A. \& Allen, D. G. Oscillations of intracellular $\mathrm{Ca}^{2+}$ in mammalian cardiac muscle. Nature 304, 735-738, https://doi.org/10.1038/304735a0 (1983).

42. Decker, K. F., Heijman, J., Silva, J. R., Hund, T. J. \& Rudy, Y. Properties and ionic mechanisms of action potential adaptation, restitution, and accommodation in canine epicardium. Am J Physiol Heart Circ Physiol 296, H1017-1026, https://doi.org/10.1152/ ajpheart.01216.2008 (2009).

43. Li, Z. et al. Improving the In Silico Assessment of Proarrhythmia Risk by Combining hERG (Human Ether-a-go-go-Related Gene) Channel-Drug Binding Kinetics and Multichannel Pharmacology. Circ Arrhythm Electrophysiol 10, e004628, https://doi. org/10.1161/CIRCEP.116.004628 (2017)

44. Champeroux, P. et al. Prediction of the risk of Torsade de Pointes using the model of isolated canine Purkinje fibres. Br J Pharmacol 144, 376-385, https://doi.org/10.1038/sj.bjp.0706070 (2005).

\section{Acknowledgements}

This work was financially supported by the National Science Foundation of China (Grant No. U1604178) to P.L.

\section{Author contributions}

P.L. and P.Z. wrote the manuscript; P.L. designed this study; P.Z. performed the simulation; P.L. and P.Z. analyzed the data.

\section{Competing interests}

The authors declare no competing interests.

\section{Additional information}

Supplementary information is available for this paper at https://doi.org/10.1038/s41598-019-55032-X.

Correspondence and requests for materials should be addressed to P.L.

Reprints and permissions information is available at www.nature.com/reprints.

Publisher's note Springer Nature remains neutral with regard to jurisdictional claims in published maps and institutional affiliations.

(c) (i) Open Access This article is licensed under a Creative Commons Attribution 4.0 International

License, which permits use, sharing, adaptation, distribution and reproduction in any medium or format, as long as you give appropriate credit to the original author(s) and the source, provide a link to the Creative Commons license, and indicate if changes were made. The images or other third party material in this article are included in the article's Creative Commons license, unless indicated otherwise in a credit line to the material. If material is not included in the article's Creative Commons license and your intended use is not permitted by statutory regulation or exceeds the permitted use, you will need to obtain permission directly from the copyright holder. To view a copy of this license, visit http://creativecommons.org/licenses/by/4.0/.

(C) The Author(s) 2019 